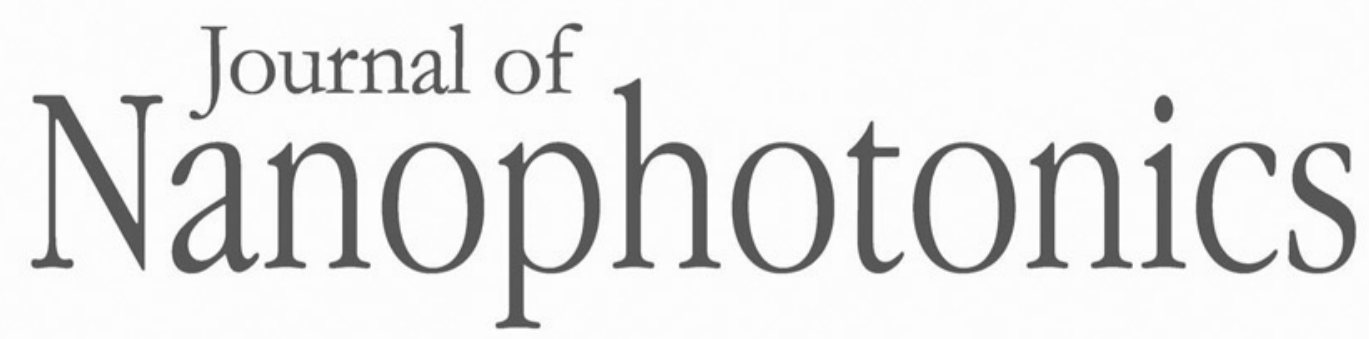

\title{
Electrical control simulation of near infrared emission in SOI-MOSFET quantum well devices
}

Michael Bendayan

Roi Sabo

Roee Zolberg

Yaakov Mandelbaum

Avraham Chelly

Avi Karsenty 


\title{
Electrical control simulation of near infrared emission in SOI-MOSFET quantum well devices
}

\author{
Michael Bendayan, ${ }^{\text {a }}$ Roi Sabo, ${ }^{\text {b }}$ Roee Zolberg, ${ }^{\text {b }}$ Yaakov Mandelbaum, \\ Avraham Chelly, ${ }^{c}$ and Avi Karsenty ${ }^{\mathrm{b}, *}$ \\ ${ }^{a}$ Rafael Advanced Defense Systems LTD, Haifa, Israel \\ ${ }^{b}$ Lev Academic Center, Department of Applied Physics/Electro-Optics Engineering, \\ Jerusalem, Israel \\ ${ }^{c}$ Bar-Ilan University, Faculty of Engineering, Ramat Gan, Israel
}

\begin{abstract}
In the race to realize ultrahigh-speed processors, silicon photonics research is part of the efforts. Overcoming the silicon indirect bandgap with special geometry, we developed a concept of a metal-oxide-semiconductor field-effect transistor, based on a silicon quantum well structure that enables control of light emission. This quantum well consists of a recessed ultrathin silicon layer, obtained by a gate-recessed channel and limited between two oxide layers. The device's coupled optical and electrical properties have been simulated for channel thicknesses, varying from 2 to $9 \mathrm{~nm}$. The results show that this device can emit near infrared radiation in the 1 to $2 \mu \mathrm{m}$ range, compatible with the optical networking spectrum. The emitted light intensity can be electrically controlled by the drain voltage $V_{\mathrm{ds}}$ while the peak emission wavelength depends on the channel thickness and slightly on $V_{\mathrm{ds}}$. Moreover, the location of the radiative recombination source inside the channel, responsible for the light emission, is also controllable through the applied voltages. (C) The Authors. Published by SPIE under a Creative Commons Attribution 3.0 Unported License. Distribution or reproduction of this work in whole or in part requires full attribution of the original publication, including its DOI. [DOI: 10.1117/1.JNP.11 $.036016]$
\end{abstract}

Keywords: Silicon-on-Insulator metal-oxide-semiconductor field-effect transistor; quantum well; simulation; optoelectronic effect; light emission; optical communication.

Paper 17019 received Mar. 1, 2017; accepted for publication Aug. 1, 2017; published online Aug. 23, 2017.

\section{Introduction}

The need for higher processing speed imposes a great technological challenge since reducing the internal distance between the processor transistors increases RF interference phenomena due to the electron-motion-induced electric field in the internal communication path. It is commonly accepted that one of the best ways to overcome these interference problems is to use optical communication instead of electrical ones since photons do not interact between them. ${ }^{1-3}$ Therefore, great efforts are conducted to obtain light-emitting Si-based devices as building blocks of integrated optical and electrical processing. ${ }^{4-7}$

Unfortunately, $\mathrm{Si}$ has an indirect bandgap that prevents electron recombination light emission. ${ }^{8}$ This is why the technology of electro-optic devices is based mainly on direct gap III-V semiconductors, such as GaAs. However, even if GaAs high-quality devices already exist, they still remain a great technological challenge to combine both $\mathrm{Si}$ - and GaAs-based blocks in the same processor chip since it is difficult to grow high-quality GaAs layers on an Si substrate. ${ }^{9}$

From the early times of microelectronic industries, microprocessors had been developed using silicon as the starting wafer material. This technology has reached high maturity, and processors can be made at very large scale production and at low cost. Therefore, it is highly preferable to find a way to obtain optical emission from a silicon-based device rather than to combine

*Address all correspondence to: Avi Karsenty, E-mail: karsenty@jct.ac.il 
Si- and GaAs-based devices since it seems that the future forecast is well oriented to silicon photonics. ${ }^{4-6,10}$ Despite silicon's indirect bandgap nature, which makes it a very poor light emitter, ${ }^{11}$ not only has electroluminescence been observed in ultrathin silicon, ${ }^{12}$ but photo-activated silicon-based devices as well as light-emitting devices have also been recently developed. ${ }^{13,14,15}$ This renewed interest in silicon photonics has top high-tech corporations investing more and more efforts into developing these kinds of technologies. ${ }^{16}$ If electro-luminescence (EL) from silicon metal-oxide-semiconductor (MOS) devices can be used for testing integrated circuits (ICs), ${ }^{17-20}$ practical silicon-emitting devices are still difficult to realize. Moreover, some methods developed to obtain silicon-based photoemission devices are not efficient enough or may induce some unacceptable device degradation through hot electrons' injection mechanism, causing threshold voltage shift, thermal emission, device lifetime reduction, and more. Therefore, it is believed that the most efficient way to obtain Si-based light-emitting devices is to use the intraband electron recombination in a quantum well.

Our recent research aims at a silicon-on-insulator (SOI)-metal-oxide-semiconductor fieldeffect transistor (MOSFET) device called MOSQWELL (MOSFET Quantum Well) for which the light emission can be expected from intra-sub band electron recombination in the silicon quantum well. ${ }^{21}$ In the past, some works described the influence of very thin layers in MOSFET devices ${ }^{22,23}$ as well as quantum-well-based photo detectors. ${ }^{24}$ Design and simulation of the present devices were conducted using the advanced COMSOL Multiphysics software package. The simulations describe the optical emission spectra in the 1 to $2 \mu \mathrm{m}$ domain (near infrared), which is the relevant optical communication band. In the present paper, these spectra will be presented as a function of the drain voltages, showing dependence between the light emission and the electric properties of the transistor, which is a necessary step toward optical communication.

\section{Device Simulation Model}

\subsection{Device Structure}

We have conducted simulations on devices with channel thicknesses varying from 2 to $9 \mathrm{~nm}$. Such a thin channel can be achieved from commercially available SOI layer $(50 \mathrm{~nm} \mathrm{Si}$ thickness in our case) wafers using a selective "gate-recessed" channel (GRC). As an example, a 4-nm thick channel device obtained by this method is presented in Fig. 1. Along with the three-dimensional (3-D) structure of the different layers shown in Fig. 1(a), a zoomed view of the GRC, included in two oxide layers (which is the quantum well structure), is presented in Fig. 1(b). The color legend enables the identification of the different material layers of the device: monocrystalline silicon in yellow, polysilicon in green, silicon oxide (both gate oxide and buried oxide) in red, silicon nitride in cyan, and aluminum contacts in blue.

\subsection{Need of an Accurate Structure and Mesh}

COMSOL software is based on finite elements calculation. Therefore, we defined the device using a high-density mesh of vertices. The high density of the mesh is needed because of the ultrathin channel layer. Because of computing and memory limitations, we had to vary the mesh density along the device so that the software will not crash and the simulation will run in reasonable time. The mesh model is shown in Fig. 2. It can be seen that the large structures are described with relatively few vertices, whereas much more vertices are needed to faithfully describe the thin layers.

\subsection{Physical Model Limitations}

Though the calculations were conducted under the semiconductor module package of COMSOL, we had to adapt the module to take into account the expected quantum effects in the ultrathin transistor channel, which can be described as a quantum well. The COMSOL software package enables postprocessing variables improved for spontaneous emission. ${ }^{25}$ These new postprocessing variables have been added, enabling the spontaneous emission spectrum to 


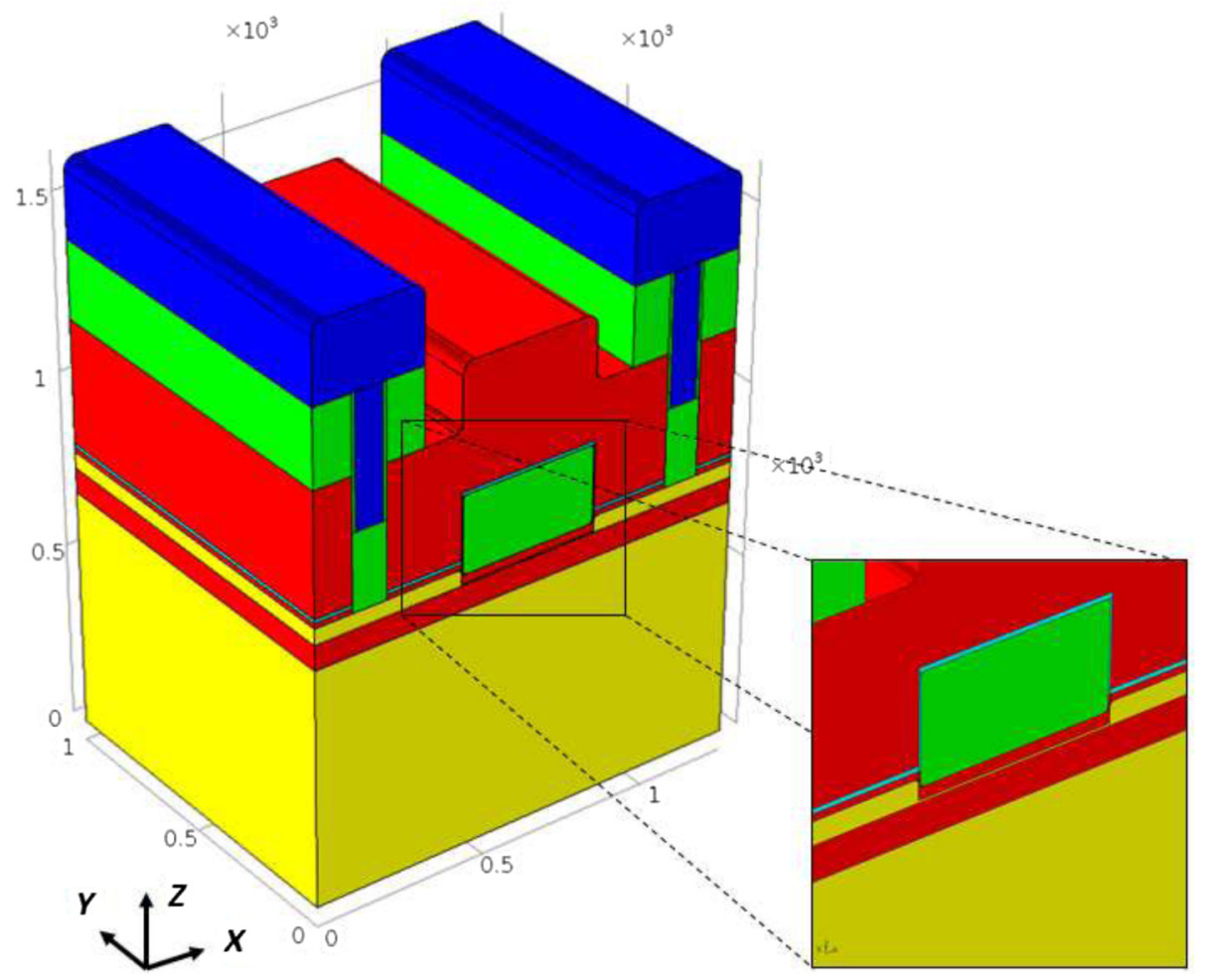

(a)

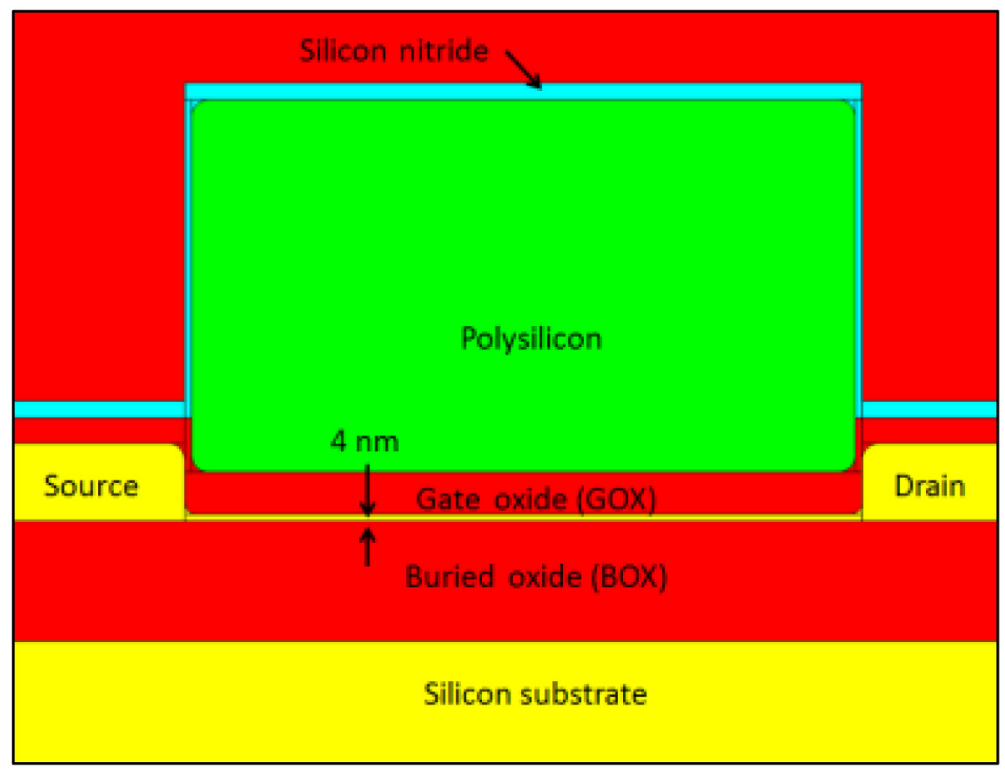

(b)

Fig. 1 Description of the MOSQWELL device structure using COMSOL multiphysics package: (a) 3-D structure. The color legend presents the different material layers: silicon (yellow), polysilicon (green), silicon oxide (red), silicon nitride (cyan), and aluminum (blue). (b) Zoomed view of the recessed channel (4-nm thickness). SOI layer is $50 \mathrm{~nm}$. Units are in $\mathrm{nm}$.

be plotted as a function of photon energy, wavelength, and frequency. Additionally, it is now possible to directly access the photon energy, wavelength, and frequency variables throughout the extra dimension that is added by the optical transitions feature, where previously these quantities needed to be calculated using an expression in terms of the angular frequency.

Previous works also showed the usage of COMSOL modules and Schrödinger-Poisson equation model solving. ${ }^{26-29}$ Indeed a two-dimensional (2-D) Poisson-Schrödinger solver is 


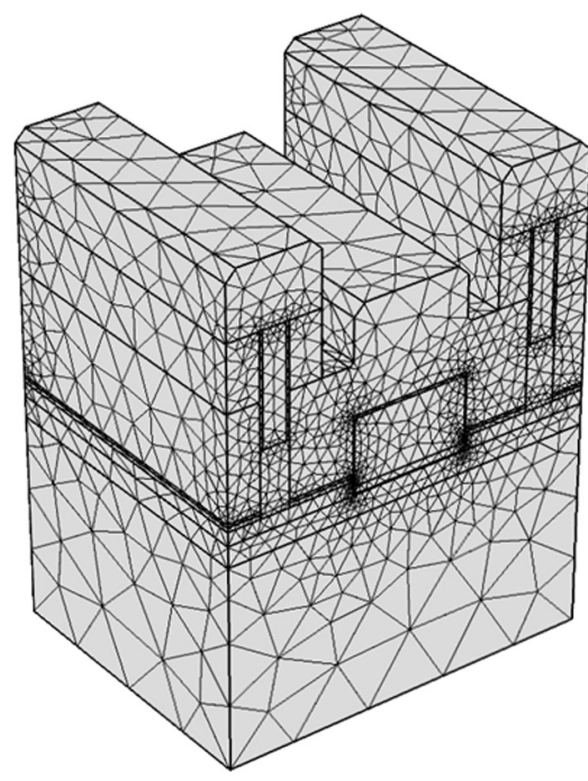

(a)

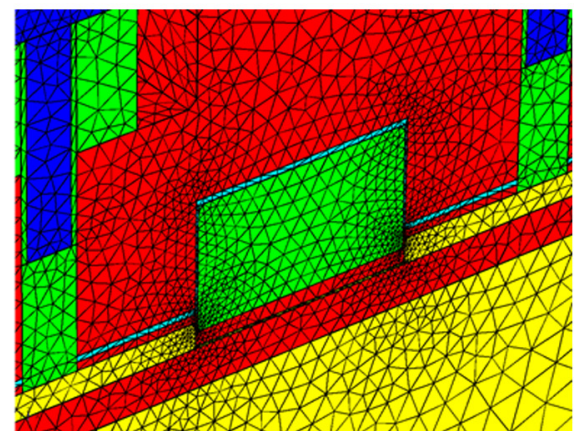

(b)

Fig. 2 Complex mesh for the COMSOL simulation of the MOSQWELL device's structure: (a) 3-D view of the dense mesh and (b) zoomed view of the device channel with more dense regions in the upper layers.

needed. It should be capable of producing a potential profile and of calculating the Eigen energy at any cross section between the drain and the source. It is of course required to obtain various sub band profiles from the drain to the source for calculating the drain current by the mode-space approach, where the transmission coefficient needs to be calculated for different sub band profiles from the drain to the source. ${ }^{27}$ By this means, we were able to calculate the light-emission intensity dependence on the drain voltage. To take into account the expected quantum effects in the ultrathin transistor channel, which can be described as a quantum well, we had to use the module in the following way. We defined the channel as a virtual semiconductor having electronic property values taken from silicon. The gaps between the inter-sub band levels were set equal to the energy level transition found from the quantum well model described in the following section. Therefore, we could not calculate the whole emission spectrum from the device, and we had to conduct the simulation for each emission line (radiative transition) separately for a given drain and gate voltages. By this means, we were able to calculate the light emission intensity dependence on the drain voltage for the whole emission spectrum. In spite of some drawbacks, the simulation will give a coherent dependence of the light emission on the drain voltage that actually determines the electron concentration along the channel and, therefore, the electric field and the electron states in the channel.

\section{Quantum Well Model}

\subsection{Emission Energies and Corresponding Wavelengths}

As a result of the momentum conservation law, the indirect bandgap nature of silicon prevents the radiative electron hole recombination. ${ }^{30}$ The minimum conduction band energy level is near the Brillouin zone edge and, therefore, has high momentum while the highest valence band energy level is at the Brillouin zone center. Since the photon having energy equal to the difference between these two energy levels should have a negligible momentum, radiative recombination due to interband charge transitions are forbidden in normal conditions. The main interest of the quantum well effect is to shift all the electron transitions at the minimum conduction band 
energy. The quantum effect will induce some energy sub bands at the same Brillouin zone point; therefore, electron sub band transitions will be allowed, and photon emission will be obtained. ${ }^{8}$

In the MOSQWELL transistor, the well is the $\mathrm{n}$-doped $\mathrm{Si}$ channel between the buried oxide layer and the gate oxide layer [Fig. 1(b)] with respective thicknesses of 75 and $25 \mathrm{~nm}$. It should be emphasized that the same Si layer serves as the recessed channel and the gates below the electrical contacts where the layer thickness is different: $4 \mathrm{~nm}$ for the channel and $50 \mathrm{~nm}$ for the source (S) and drain (D) contacts. While quantum effects are expected in the channel, the silicon thickness in the drain and source regions is large enough so that quantum effects are negligible. Let us consider that the silicon layer is confined in the $z$ direction (the growth direction) between the $\mathrm{SiO}_{2}$ layers and the layer has variable width in the $x$ direction. In the $y$ direction, the layer can be considered infinite. We can assume that the electron motion in the $x$ direction is free, although there is a small potential step at the interfaces between the gate and the channel. Therefore, the electrons behave as a 2-D free electron gas in the $x y$ plane. The quantum-well-induced energy levels $E$ are then obtained by solving the one-dimensional (1-D) Schrödinger equation:

$$
E \psi(z)=-\frac{\hbar^{2}}{2 m_{z}} \frac{d \psi}{d z}+V(z) \psi(z)
$$

where $V(z)$ is the potential energy in the confined ( $z$ ) direction, $m_{z}$ is the effective mass of the electron, and $\psi(z)$ is the electron wave function. The energy band diagram of the quantum channel is described in Fig. 3. The $\mathrm{SiO}_{2}$ bandgap is $8.9 \mathrm{eV}{ }^{31}$ and the energy difference between the conduction band of the $\mathrm{Si}$ well and the $\mathrm{SiO}_{2}$ barrier (the barrier height) is $3.3 \mathrm{eV} .{ }^{31}$ We assume that the electron effective mass in the $\mathrm{SiO}_{2}$ barrier is the free electron mass $m_{0} \cdot{ }^{31,32} \mathrm{In}$ the $\mathrm{Si}$ well, the effective electron mass is the transverse conduction band electron effective mass $0.2 m_{0}{ }^{33}$ near the conduction minimum since the $\mathrm{Si}$ is grown in the (100) direction.

The relative intensity of the light emission of the given transition between sub band levels $i$ and $f$, which is related to the transition probability per unit of time between confined levels, is calculated from the transition probability per unit of time $\Gamma_{i \rightarrow f}$ given by the Fermi golden rule

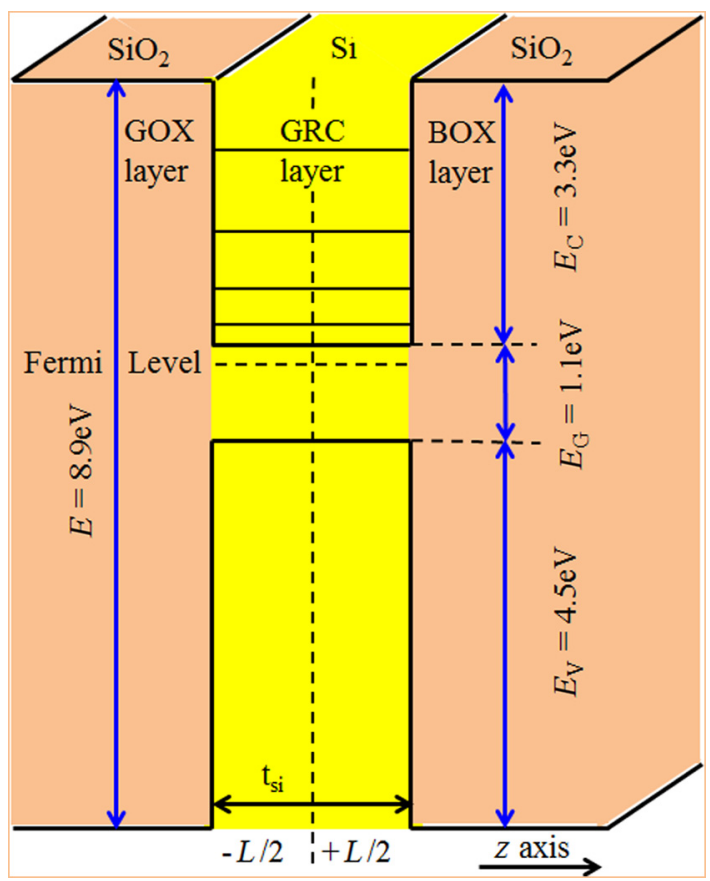

Fig. 3 Schematics of the energy band diagram (conduction and valence bands) of the quantum well channel structure, for a given channel thickness $L$. In our case, only the quantum sublevels in the conduction band are relevant since we consider an $n$-MOSFET device. 


$$
\Gamma_{i \rightarrow f}=\frac{2 \pi}{\hbar}\left|\psi_{f}\right| H\left|\psi_{i}\right|^{2} \rho
$$

where $H$ is the electric dipole transition operator q.r (the perturbing Hamiltonian that is responsible for the photon emission by electron recombination), $\rho$ is the density of final states, and $\psi_{i}$ and $\psi_{f}$ are the initial and final electron state wave function, respectively. Because of the asymmetric nature of the transition operator, the main electron transitions can only be between states of opposite symmetry.

The 1-D Schrödinger Eq. (1) can be solved numerically to obtain the different energy levels in the well. For the well thicknesses $L$ that we are dealing with $(2,3, \ldots 9 \mathrm{~nm})$, the energy levels are shown in Table 1 for the even and the odd solutions of the equation. The allowed transition energy level emission energies and wavelengths corresponding to the optical communication domain (typically 1 to $2 \mu \mathrm{m}$ ) and beyond the optical absorption threshold of silicon are also shown in the table.

\subsection{Simulated Spectrum Modeling}

In addition to the single line calculation, the overall emission spectra are obtained by combining the different lines in a single spectrum using a Lorentzian broadening

$$
S(\lambda)=\frac{1}{\pi} \sum_{i=1}^{N_{\text {peaks }}} \Gamma_{j \rightarrow k} \frac{\alpha_{L}}{\left(\lambda-\lambda_{i}\right)^{2}+\alpha_{L}^{2}},
$$

where $\alpha_{L}$ is the Lorentzian full width at half maximum and $\lambda_{i}$ is the transition photon wavelength of the $i$ 'th peak among the total number of peaks $N_{\text {peaks }}$ and corresponding to the allowed transition between the $j$ and $k$ states of opposite symmetry. The broadening is due to many reasons such as nonuniformity, temperature, and interface roughness. We assumed an full width of half maximum $\alpha$ of $0.05 \mathrm{eV}$, which roughly corresponded to the width of Si-based device electroluminescence at room temperature. ${ }^{15}$

Table $1 \mathrm{Si}$ well sublevel energies $(\mathrm{eV})$ in the conduction band obtained for several well thicknesses of up to $4 \mathrm{~nm}$. Emission energies $(\mathrm{eV})$ and corresponding wavelengths $(\mu \mathrm{m})$ are also summarized.

\begin{tabular}{lcccc}
\hline \hline $\begin{array}{l}\text { Si well } \\
\text { thickness } \\
L(\mathrm{~nm})\end{array}$ & $\begin{array}{c}\text { Even solution energy } \\
\text { levels }(\mathrm{eV})\end{array}$ & $\begin{array}{c}\text { Odd solution energy } \\
\text { levels }(\mathrm{eV})\end{array}$ & $\begin{array}{c}\text { Emission energies } \\
(\mathrm{eV})\end{array}$ & $\begin{array}{c}\text { Emission } \\
\text { wavelengths }(\mu \mathrm{m})^{\mathrm{a}}\end{array}$ \\
\hline 2 & $0.2,2.3$ & 0.95 & 0.75 & 1.65 \\
3 & $0.11,1.2$ & $0.52,2.2$ & $0.68,1$ & $1.82,1.24$ \\
4 & $0.076,0.74,2.2$ & $0.32,1.4,3.2$ & $0.66,0.8,1$ & $1.88,1.55,1.24$ \\
5 & $0.045,0.51,1.45,2.94$ & $0.21,0.91,2.1$ & $1.24,0.86,0.65$, & $1,1.44,1.9,1.47$ \\
6 & $0.039,0.37,1.1, .21$ & $0.16,0.64,1.5,2.8$ & $0.94,1.13,0.7$ & $1.32,1.09,1.77$ \\
7 & $0.03,0.28,0.8,1.6,2.6$ & $0.12,0.49,1.2,2.1$, & $0.68,1.11,1.17$, & $1.82,1.11,1.05$, \\
& & 3.3 & $0.92,0.7$ & $1.34,1.77$ \\
8 & $0.023,0.22,0.62,1.24$, & $0.095,0.39,0.9,1.6$, & $1.14,0.85,0.87$, & $1.08,1.46,1.42$, \\
& 2.1 & 2.6 & $0.68,1.2,0.98$ & $1.82,1.03,1.26$ \\
& $0.0186,0.175,0.495$, & $0.077,0.315,0.72$, & $0.913,0.675,0.94$, & $1.36,1.83,1.32$, \\
9 & $0.99,1.665,2.515$ & $1.305,2.07,3$ & $1.13,0.81,1.21$ & $1.09,1.53,1.02$ \\
\hline \hline
\end{tabular}

${ }^{\mathrm{a} C}$ Calculations are limited to $2 \mu \mathrm{m}$ for practical considerations to match the optical communication spectra. 


\section{Simulation Results}

\subsection{Electrical Output Characteristics}

Prior to running the optical simulations, we had to simulate the electrical characteristics using a COMSOL multiphysics package for the device having a 4-nm thick channel value. The aim of those simulations was to assure that the device structure is behaving like a regular MOSFET transistor. Output characteristics, i.e., drain current versus drain voltage $\left(I_{\mathrm{ds}}-V_{\mathrm{ds}}\right)$ with gate voltage $V_{\mathrm{g}}$ stepping from 0 to $4 \mathrm{~V}$ were simulated (Fig. 4). As expected from an MOSFET transistor, the saturation regime is reached after the linear one for each $V_{\text {gs }}$ voltage of the output curves. The avalanche breakdown zone was also empirically described by exponential trend (as inserted in Fig. 4). We can extract the "activation" energy $E_{\mathrm{a}}$ of the avalanche process, defined by the exponential term $e^{\mathrm{qVds} / \mathrm{Ea}}$, by taking the reciprocal value of the fitting slope; we get $E_{\mathrm{a}}=0.35 \mathrm{eV}$. Thus, this satisfying transistor behavior acts as a functionality check of the electrical simulation part.

\subsection{Optical Emission Spectra}

\subsubsection{Emission wavelength as function of the channel thickness}

Once the device is defined in COMSOL and the allowed transitions have been calculated, we can simulate for a given transition the emission spectra for different channel thicknesses. The calculations were made for channel thicknesses of 2 to $9 \mathrm{~nm}$ to evaluate the relevant light-emitting transitions between the allowed intersub band energy levels as depicted in Table 1. The simulated graph results (Figs. 5-8) present the intensity in arbitrary units (a. u.) of the light emission, as a function of the wavelength (emission spectrum) of up to $2 \mu \mathrm{m}$. Note that an emission wavelength below $1.1 \mu \mathrm{m}$ is not considered because of the silicon band-to-band optical absorption. From Fig. 5, we can see that a thickness of $3 \mathrm{~nm}$ does not give better emission in the desired wavelength range, since most of its light emission is at a greater wavelength. This is because, for this larger thickness, the energy of transition is lower than for the 2-nm thickness case. Despite the fact that this larger thickness allows an additional electron-confined energy level, this level is too high to allow a transition in the required wavelength domain. By further increasing the channel thickness to $4 \mathrm{~nm}$ and higher, the energy levels go even lower, which allows an additional transition in the required (near IR) domain, as can be seen in Figs. 5-8.

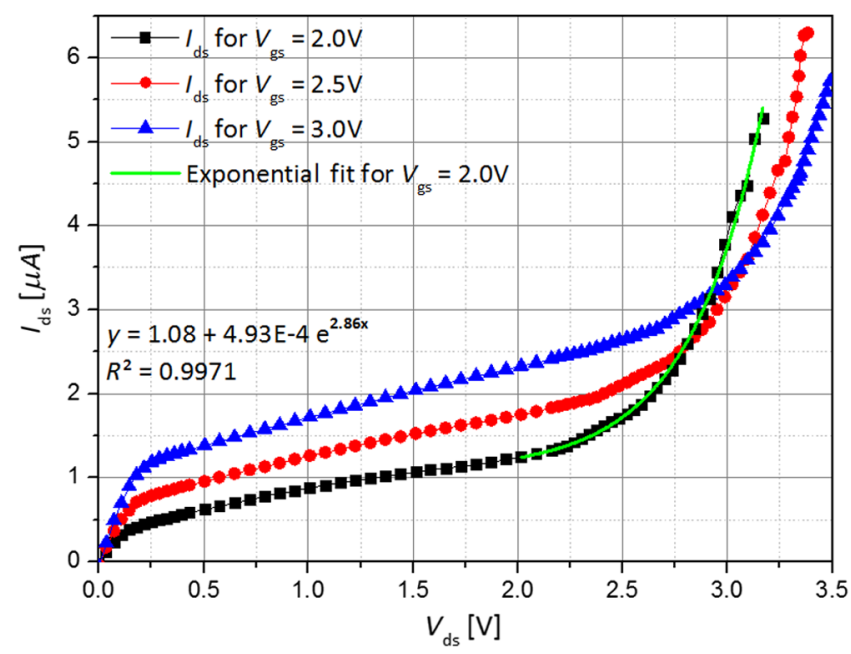

Fig. 4 Simulated characteristics of an MOSQWELL device having a channel thickness of $4 \mathrm{~nm}$ at breakdown regime. 
Bendayan et al.: Electrical control simulation of near infrared emission...

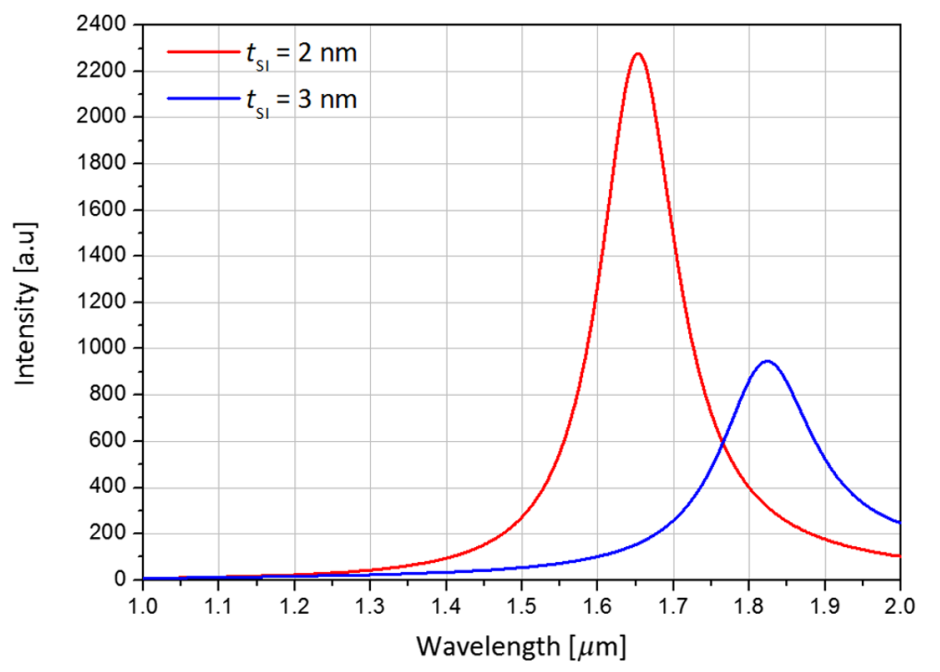

Fig. 5 Simulated light-emission spectra for device with channel thickness of 2 and $3 \mathrm{~nm}$.

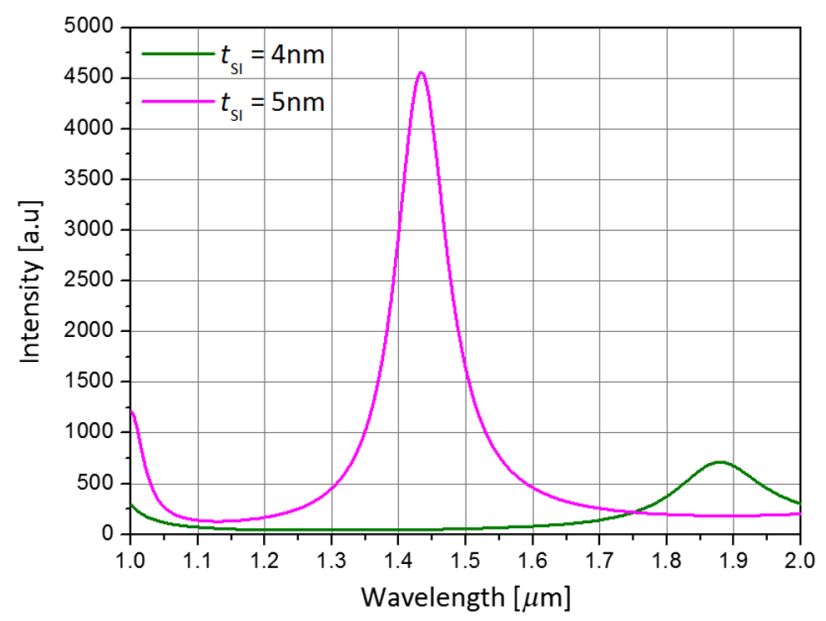

Fig. 6 Simulated light-emission spectra for device with channel thickness of 4 and $5 \mathrm{~nm}$.

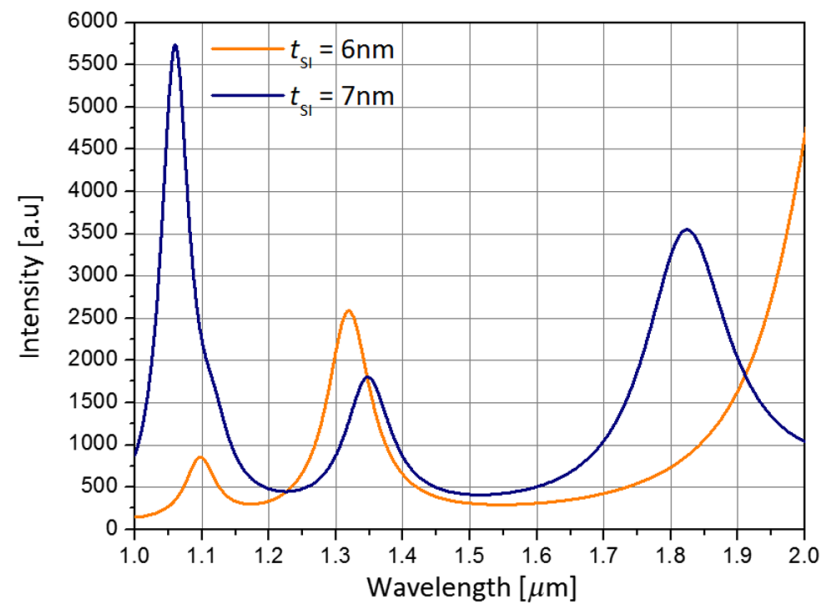

Fig. 7 Simulated light-emission spectra for device with channel thickness of 6 and $7 \mathrm{~nm}$. 


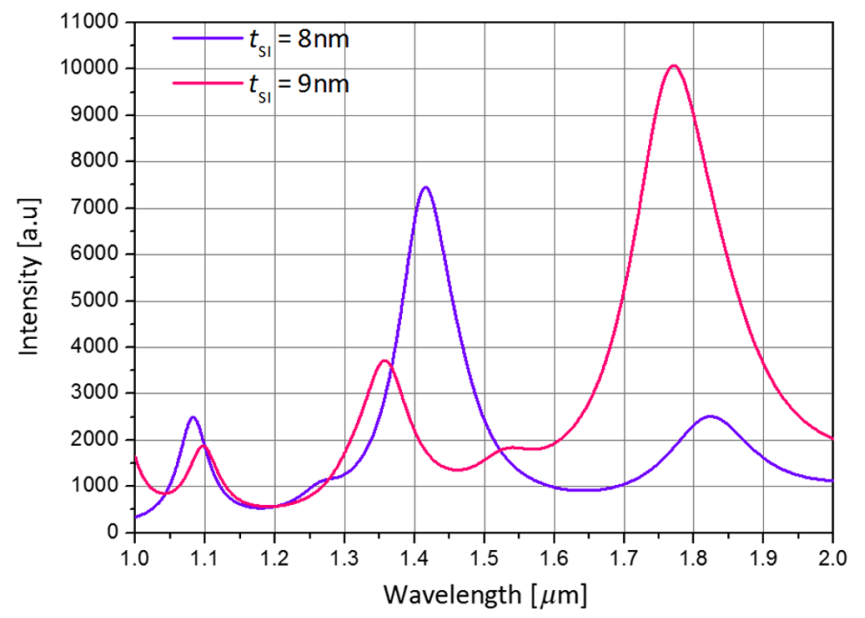

Fig. 8 Simulated light-emission spectra for device with channel thickness of 8 and $9 \mathrm{~nm}$.

\subsubsection{Control of the light intensity as a function of the drain $V_{d s}$}

As a worst case test, we selected the 4-nm thick channel device because of its relatively small peak intensity. The emission spectrum was simulated for the first emission peak located at $1.88 \mu \mathrm{m}\left(0.66 \mathrm{eV}\right.$ energy transition) and for different electrical conditions ( $V_{\mathrm{gs}}$ kept as $2 \mathrm{~V}$ and $V_{\mathrm{ds}}$ varying from 2.34 to almost $3 \mathrm{~V}$ to get in the avalanche breakdown regime, as shown in Fig. 9). As expected, the intensity increases steeply with $V_{\mathrm{ds}}$, indicating that the more hot electrons are present in the channel, the stronger the light emission. More precisely, it appears that the light emission has a threshold electrical condition on $V_{\mathrm{ds}}$, which is connected to the breakdown regime.

The emitted light intensity is directly related to the emitted photon quantity. Since a photon is the result of one electron recombination, the more electrons there are in the channel, the more photons will be emitted and therefore the greater the light intensity. In the transistor channel, the electron quantity is exponentially related to the drain voltage, so should be the light intensity. In Fig. 10, we can fit the intensity dependence of each spectral line on $V_{\mathrm{ds}}$, by an exponential dependence like $e^{\mathrm{qVds} / \mathrm{Ea}}$. The extraction of the activation energy $E_{\mathrm{a}}$ as the reciprocal of the slope is $1 / 7.39=0.13 \mathrm{eV}$. Note that it has the same order of magnitude of those found for the avalanche breakdown (Fig. 4).

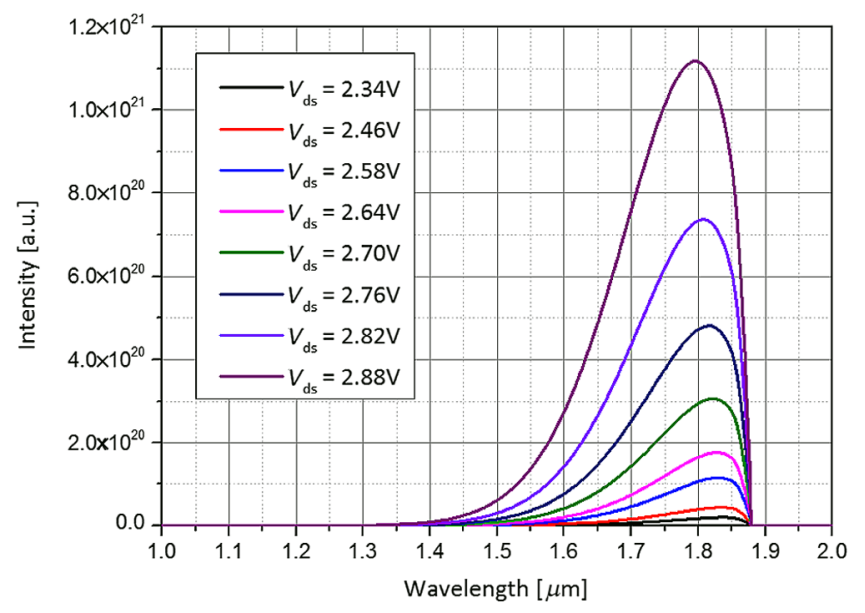

Fig. 9 Intensity of the emission spectra for several $V_{\text {ds }}$ values simulated for intersub bandgap of $0.66 \mathrm{eV}\left(1.88-\mu \mathrm{m}\right.$ wavelength). Device is $4-\mathrm{nm}$-thick channel device $\left(V_{\mathrm{gs}}=2 \mathrm{~V}\right)$. 
Bendayan et al.: Electrical control simulation of near infrared emission...

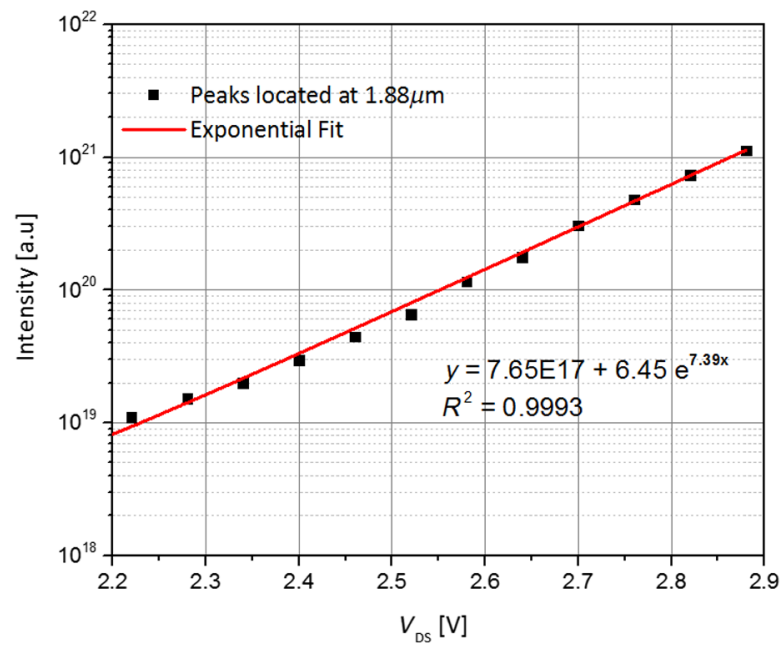

Fig. 10 Intensity dependence of the $1.88 \mu \mathrm{m}$ emission peak for 4-nm thick channel device at $V_{\mathrm{gs}}=2 \mathrm{~V}$.

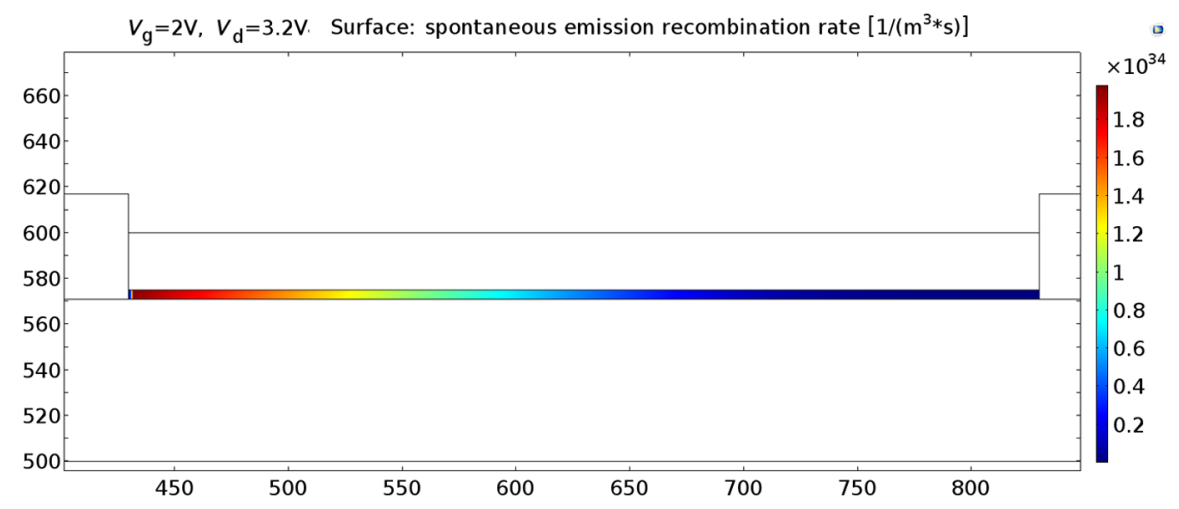

Fig. 11 Distribution of the radiative recombination rate along the silicon channel for $V_{\mathrm{ds}}=3.2 \mathrm{~V}$. Horizontal scale is in $\mathrm{nm}$.

\subsubsection{Location of the light emission in the channel}

As part of the analysis of the device behavior and following previous setup conditions, it was important to simulate and understand not only the intensity of the spontaneous light emission $(1.88 \mu \mathrm{m})$ from the silicon channel $(4 \mathrm{~nm})$ but also its location and spatial distribution. Assuming that the intensity of the light emission due to the transitions between the quantum levels is proportional to the spontaneous recombination rate, we simulated the distribution of this rate in the channel, as shown in Fig. 11. The maximum value is located close to the channel edge and decays rapidly along the channel.

\section{Analysis and Discussion}

As mentioned above, the aim of this analysis is to demonstrate the existing coupling of optical properties to the device quantum structure and electrical parameters, so, respectively, the wavelength of the emission is a function of the channel thickness, and the intensity of the peak is controllable by the drain applied voltage.

\subsection{Electrical Study}

Regarding the electrical characteristics shown in the avalanche breakdown regime, the electrons are "pumped" to higher energy sublevels of the conduction band. From this high-energy state, 
the electrons can relax to lower sublevels and can emit a photon. Since one photon is emitted by one electron recombination, the emitted light intensity, which is proportional to the number of emitted photons (for a given wavelength), is proportional to the current passing through the channel. As seen in Figs. 9 and 10, the light intensity is indeed increasing in a same kind of exponential trend with $V_{\mathrm{ds}}$ like in the avalanche breakdown regime. In spite of the fact that we work in the avalanche breakdown regime, we remain in a relatively low $V_{\mathrm{ds}}$ range (up to $2.6 \mathrm{~V}$ ), so we can assume that there will not be a strong degradation of the device functionality.

\subsection{Adjustment of the Wavelength as a Function of the Channel Thickness}

As shown above and as expected due to the number of transitions, the thicker the channel is, the higher the number of peaks is and the higher the intensity is (Figs. 5-8). This allows us to choose the best thickness to obtain the higher throughput in the required wavelength and the required bandwidth. For example, if we are interested in monochromatic emission, we may choose thin layers ( 2 or $3 \mathrm{~nm}$ ). We can therefore introduce a new transistor descriptive parameter that sets the emitted wavelength.

\subsection{Control of the Light Intensity as a Function of the Drain $V_{d s}$}

As for the light-intensity control, the results may enable some preliminary forecast of the expected highest intensity as a function of the thickness for a defined window, in our case, the 1 to $2 \mu \mathrm{m}$ range. Moreover, as the drain voltage controls the light intensity, we could reasonably assume that an electrical signal applied at the gate can also modulate the light emission. From these preliminary simulated results, we can expect that, by coupling Si waveguides to the channel, the optical signal can be transmitted to a detector coupled to another transistor. This is actually the elementary communication operation between two elementary devices where this communication is equivalent to the electron communication between devices or blocks. The main difference here is that there is no more $\mathrm{RC}$ delay limiting the electron motion and no cross talk between the devices. Since there is no cross talk, the devices can be closer and the modulation speed can get higher without tampering with the processor work.

\subsection{Usage and Possible Applications}

The need for future generations of very large-scale integrated circuits working at $10 \mathrm{GHz}$ frequencies and above is a challenge that CMOS technology has tried to reach for several decades. In such frequencies, the signal propagation delay on a chip and a circuit board, as well as signal cross talk, impose severe limitations on system design and performance. A proposed solution to this problem is to move to optical signal transmission in the critical paths. The merging of the microelectronics with communication in general and with optical communication in particular vigorously pushes the efforts to realize both electronic and electro-optic functions on the same silicon chips. The devices presented in this study allow this kind of communication. This is further motivated by the limitations of the metal wiring on a chip and a PC board to transfer the electronic signals in the $10 \mathrm{GHz}$ range and above. Moreover, failure mechanisms in metal interconnect, such as self-heat and electromigration, can be prevented in the case of optical communication between blocks when using optical emission through silicon waveguides.

In our preliminary study, we have emphasized that QW MOSFET processing is compatible with standard CMOS technology. Moreover, SOI CMOS on SOI wafers is already a commercial process, and the expected light emission at selected wavelengths, in correlation with the silicon thickness in the transistor, may lead to the development of modified SOI CMOS technology that will include both standard CMOS transistors for the performance of the electronic functions and quantum well transistor devices for optical communication. We are aware of the very low emission intensity that may be emitted by the device; however, this concern should not be detrimental since the device is not intended to serve as a laser emitter but rather as an electro-optic modulator. In other words, the aim of this device is to act as a local emitter of IR radiation, which may be 
electrically modulated and eventually detected and amplified by other detectors in its close neighborhood, using a built-in waveguide. Assuming that we can build such a light-emitting transistor, we can obtain a device that can convert ultrahigh-frequency electric signal to an optical signal, which in turn can propagate without any coupling or radiative effects. This may dramatically increase the processor work frequency.

\section{Conclusions}

In this article, we presented a new nanoscale MOSFET Quantum Well (MOSQWELL) transistor based on a GRC quantum-channel structure. The model of the device is described, and simulation results are presented. Optical simulations show promising light-emission features (both in intensity and in wavelength) adapted to optical communication. On the other hand, electrical simulation results were found promising toward future measurements on devices. To get a series of measurements, several thicknesses would be processed and characterized. The model presents several expected values of energy levels, which can enable light-emitting mechanism, and shows the control of the drain voltage on the emission intensity in the breakdown avalanche regime.

\section{References}

1. N. Savage, "Linking chips with light," IEEE Spectrum, 2015, http://spectrum.ieee.org/ semiconductors/optoelectronics/linking-chips-with-light.

2. J. Mason, "Development of on-chip optical interconnects for future multi-core processors," 2008, http://www.smartdatacollective.com/jackmason/22794/development-chip-opticalinterconnects-future.

3. A. W. Bogalecki and M. du Plessis, "Design and manufacture of quantum-confined Si light sources," S. Afr. Inst. Electr. Eng. 101(1), 11-16 (2010).

4. L. Pavesi, "Will silicon be the photonic material of the third millennium?" J. Phys.: Condens. Matter 15, R1169 (2003).

5. D. Thomson et al., "Roadmap on silicon photonics," J. Opt. 18, 073003 (2016).

6. J. Faist, "Silicon shines on," Nature 433, 691-692 (2005).

7. M. Lipson, "Guiding, modulating, and emitting light on silicon-challenges and opportunities," J. Lightwave Technol. 23(12), 4222-4238 (2005).

8. N. Ashcroft and N. Mermin, Solid State Physics, Saunder College, Philadelphia (1976).

9. Y. B. Bolkhovityanov and O. Pchelyakov, "GaAs epitaxy on Si substrates: modern status of research and engineering," Phys. Uspekhi 51, 437-456 (2008).

10. A. T. Fiory and N. M. Ravindra, "Light emission from silicon: some perspectives and applications," J. Electron. Mater. 32(10), 1043-1051 (2003).

11. S. Coffa, "Light from silicon," IEEE Spectrum, 2005, http://spectrum.ieee.org/semiconductors/ processors/lightfromsilicon.

12. S. Saito et al., "Electro-luminescence from ultra-thin silicon," Jpn. J. Appl. Phys. 45(27), L679-L682 (2006).

13. A. Zev et al., "Nanoscale silicon-on-insulator photo-activated modulator building block for optical communication," IEEE Photonics Technol. Lett. 28(5), 569-572 (2016).

14. W. L. Ng et al., "An efficient room-temperature silicon-based light-emitting diode," Nature 410, 192-194 (2001).

15. H. Rong et al., "An all-silicon Raman laser," Nature 433(20), 292-294 (2005).

16. S. Koehl, V. Krutul, and M. Paniccia, "Continuous silicon laser," Intel White Paper (2005).

17. J. A. Kash and J. C. Tsang, "Hot luminescence from CMOS circuits: a picosecond probe of internal timing," Phys. Stat. Sol. (B) 204, 507-516 (1997).

18. J. C. Tsang and J. A. Kash, "Hot luminescence from CMOS circuits: a picosecond probe of internal timing," Appl. Phys. Lett. 70(7), 889-891 (1997).

19. J. C. Tsang, J. A. Kash, and D. P. Vallett, "Optical tools for measuring timing and switching in silicon ICs: Status and future challenges," LEOS Newsl. 15(2), 3-5 (2001). 
20. S. K. Kurinec et al., "Utilization of electroluminescence from avalanche p-n junctions for optical testing of silicon integrated circuits," in Proc. IEEE Micro-Electronics 8th Symp. (1989).

21. M. Bendayan, A. Chelly, and A. Karsenty, "Modeling and simulations of MOSQWell transistor future building block for optical communication," in Proc. IEEE Int. Conf. on the Science of Electrical Engineering (ICSEE), Eilat, pp. 1-5 (2016).

22. J. Walczak and B. Majkusiak, "Scattering mechanisms in MOS/SOI devices with ultrathin semiconductor layers," J. Telecommun. Inf. Technol. 1, 39-44 (2004).

23. E. Gornik and D. C. Tsui, "Voltage-tunable far-infrared emission from Si inversion layers," Phys. Rev. Lett. 37, 1425-1428 (1976).

24. L. Liu et al., "Highly efficient metallic optical incouplers for quantum well infrared photodetectors," Nat. Sci. Rep. 6, 30414 (2016).

25. "Comsol multiphysics 5.1 release highlights," Indirect Optical Transitions, https://www. comsol.com/release/5.1/semiconductor-module.

26. A. J. Kalinowski, "Quantum mechanics applications using the time dependent Schrödinger equation in COMSOL," in Proc. of the 2015 COMSOL Conf., Boston (2015).

27. S. Jahangir and Q. D. M. Khosru, "A numerical model for solving two-dimensional Poisson-Schrodinger equation in depletion all around operation of the SOl four gate transistor," in IEEE Int. Conf. of Electron Devices and Solid-State Circuits (2009).

28. M. V. Kisin, "Modeling of the quantum well and cascade semiconductor lasers using 8-band Schrödinger and Poisson equation system," in Proc. of 2007 COMSOL Conf., Boston, pp. 489-493 (2007).

29. M. V. Kisin and H. S. El-Ghoroury, "Modeling of III-nitride multiple quantum well light emitting structures," IEEE J. Sel. Top. Quantum Electron. 19(5), 1-10 (2013).

30. B. E. A. Saleh and M. C. Teich, "Fundamentals of photonics," in Chapter 15, Photons in Semiconductors, John Wiley \& Sons, Inc., Hoboken, New Jersey (1991).

31. I. Filikhin et al., "Electronic and level statistics properties of $\mathrm{Si} / \mathrm{SiO}_{2}$ quantum dots," Phys. E: Low-Dimens. Syst. Nanostruct. 42, 1979-1983 (2010).

32. M. Dragosavac et al., "Electron effective mass in ultrathin oxide silicon MOSFET inversion layers," Semicond. Sci. Technol. 20, 664-667 (2005).

33. B. Van Zeghbroeck, "Principles of electronic devices," in Detailed Description of the Effective Mass, University of Colorado Boulder, Boulder, Colorado (2011).

Michael Bendayan received his $\mathrm{PhD}$ at the Technion Institute. He is part of the research team under the supervision of Dr. Avi Karsenty. He is now a professional fellow at Rafael Advanced Defense Systems Ltd.

Roi Sabo received his BSc degree in the Department of Physics/Electro-Optics Engineering at the Lev Academic Center. He is part of the research team under the supervision of Dr. Avi Karsenty. He now works in the high-tech industry as an electro-optical engineer.

Roee Zolberg received his BSc degree in the Department of Physics/Electro-Optics Engineering at the Lev Academic Center. He is part of the research team under the supervision of Dr. Avi Karsenty. He now works in the high-tech industry as an electro-optical engineer.

Yaakov Mandelbaum received his BA and MA degrees in mathematics from the University of Pennsylvania, in 1993 and 1995, and was part of the Applied Mathematics Program at the Massachusetts Institute of Technology (1993-1995). He completed his MSc degree at Racah Institute of Physics (1998-2001). After working in QLight Nanotech (2011-2013) and QuantUp (2014-2015), he joined the Physics/Electro-Optics Department at the Lev-AcademicCenter (2013). He has started his PhD studies under the supervision of Dr. Karsenty and Prof. Zalevsky.

Avraham Chelly received his MSc degree in material science from the Université Louis Pasteur, France, in 1992 and his $\mathrm{PhD}$ in solid state physics from the Université de Haute Alsace, France, in 1997. He held a postdoctoral position at Hebrew University of Jerusalem, Israel. He worked as 
a research engineer at the Microelectronics Laboratory in 2004, and he moved to Bar-Ilan University, where he established the Advanced Semiconductor Devices Laboratory. He is involved in nanoscale electro-optics devices' research and lectures.

Avi Karsenty received his MSc degree and $\mathrm{PhD}$ in applied physics and material science (microelectronics and electro-optics) from Hebrew University of Jerusalem in 1996 and 2003, respectively. After 22 years in high-tech industries, part of which with Intel Electronics Corporation (1995-2011), today, he is the head of the Physics/Electro-Optics Engineering Department and of the Excellence Empowerment Program at the Lev Academic Center. He has been awarded 38 awards and is a senior member of IEEE and OSA. His major research field is quantum coupled devices. 